Horizons philosophiques

\title{
Je ne joue plus. Réflexion sur l'anthropologie augustinienne
}

\section{Christian Talin}

Volume 10, numéro 1, automne 1999

Écritures et confessions

URI : https://id.erudit.org/iderudit/801110ar

DOI : https://doi.org/10.7202/801110ar

Aller au sommaire du numéro

Éditeur(s)

Collège Édouard-Montpetit

ISSN

1181-9227 (imprimé)

1920-2954 (numérique)

Découvrir la revue

Citer cet article

Talin, C. (1999). Je ne joue plus. Réflexion sur l'anthropologie augustinienne. Horizons philosophiques, 10(1), 107-128. https://doi.org/10.7202/801110ar d'utilisation que vous pouvez consulter en ligne.

https://apropos.erudit.org/fr/usagers/politique-dutilisation/ 


\section{JE NE JOUE PLUS. RÉFLEXION SUR L'ANTHROPOLOGIE AUGUSTINIENNE}

"Et direxi me ad me et dixi mihi : "Tu quis es?"

Et respondi : "Homo". Et ecce corpus et anima in me mihi praesto sunt, unum exterius et alterum interius".

(S. Aureli Augustini, Confessionum, liber X, VI, 9.)

Pour Emmanuel Mounier, la pensée existentielle se caractérise comme "une réaction de la philosophie de l'homme contre l'excès de la philosophie des idées et de la philosophie des choses ${ }^{1}$ ". Entre les stoïciens et saint Bernard, saint Augustin est l'une des racines de "l'arbre existentialiste". Une (re)lecture des Confessions conforte ce jugement : cette philosophie de l'existence questionne l'homme en situation.

L'intériorisation augustinienne passe par les étapes de la vie. Chacun sait que les Confessions incluent trois niveaux : confession de la vie du futur saint, confession de sa foi et confession de sa louange à Dieu ..$^{2}$ Nous privilégierons le vécu, donc nous nous y "limiterons". Cet ouvrage est, sans conteste, le témoignage de son itinéraire vers Dieu dans lequel s'entrecroisent la théologie et la mystique, la philosophie et la psychologie. Sensible à l'ouverture à l'autre, saint Augustin fournit une source de réflexion tant aux philosophes qu'aux psychanalystes. Si le projet semble austère, ce récit à la première personne (dans lequel il tutoie Dieu) invite toutefois le lecteur à un rapprochement, voire à une identification. En effet, il est difficile de n'être point sensible au cheminement d'une conscience, à la richesse et la simplicité de la description de l'expérience vécue, celle d'une singularité qui porte en elle l'universalité de la condition humaine.

Prendre les Confessions comme le paradigme de l'intersubjectivité serait sans doute excessif. Elles n'en demeurent pas moins une œuvre d'inspiration prépsychologique, voire préanalytique, en tant que discours positif sur la psychologie de l'enfant. Des psychanalystes ont commenté les

1. Emmanuel Mounier, Introduction aux existentialismes, Paris : Éditions Denoël, 1946, rééd. Paris : Éd. Gallimard, coll. "ldées", n 14, 1969, p. 8-9.

2. André Mandouze, Saint Augustin. L'aventure de la raison et de la grâce, Paris : Éditions Études augustiniennes, 1968, p. 47. Voir aussi Jean-Marie Le Blond, Les Conversions de saint Augustin, Paris, Éditions Aubier-Montaigne, coll. "Théologie», 1950. 
expériences du Moi dans la vie de saint Augustin. Signalons, par exemple, l'article du professeur Jacques Chomarat ${ }^{3}$, qui porte sur l'historique de ses relations avec sa mère dominatrice, et divers commentaires de Jacques Lacan, les principaux ayant cependant trait au langage.

En dépit de l'écart entre les philosophies du cogito et la psychanalyse, et l'ignorance de la distinction entre le Je-sujet et le Je-Moi, le premier pensant dans "un autre lieu» dont le second est exclu, c'est-à-dire le Je de l'énonciation et le Je de l'énoncé dans le discours de l'analysant, nous privilégierons la perspective analytique fécondée par l'apport de la philosophie et de la logique pour cerner, au fil des âges, les étapes existentielles qui, du jeu au Je, forment un être, aussi homo sapiens que homo ludens. De la prime enfance, égocentrique et éminemment ludique, en passant par les jeux et les expériences de l'adolescence, on s'achemine, du plus bas degré de l'être au plus haut, vers l'âge adulte où le sujet authentique est capable d'accéder à une conscience philosophique du monde ainsi qu'à Dieu.

Après une action de grâces rendue à Dieu, les Confessions décrivent les étapes de la vie humaine, autrement dit la construction d'un être ou théorie du sujet. À chaque âge de l'existence : nourrisson, bébé, enfant, etc., saint Augustin consigne et commente une série d'observations qui permettent de découvrir les notions complémentaires d'identité et d'altérité. Le jeu pour le Je sera le fil conducteur que nous suivrons au cours des premiers livres des Confessions jusqu'au Je mûr du livre XI.

\section{Le concept d'altérité et la formation du Je}

II n'existe pas d'homme naturel présocial, pense Aristote, qui définit I'homme comme un «animal politique 4 ". La polis ou société est, par nature, à la fois la matrice de l'humanité et la fin qui accomplit les potentialités de la nature humaine : I'homme n'est lui-même qu'uni aux autres; la parole sert de lien dans la communauté. 5 Examinons brièvement le concept clef

3. Voir "Les Confessions de saint Augustin", Revue française de psychanalyse, publication officielle de la Société psychanalytique de Paris, Société constituante de l'Association psychanalytique internationale, Paris : Éditions PUF, tome LII, $n^{\circ} 1 / 1988$, p. 153-174.

4. Voir Les Politiques, traduction inédite par Pierre Pellegrin, Paris : Éd. Flammarion, $n^{\circ} 490,1990 ; 2^{e}$ éd. revue et corrigée, 1993, livre I, chap. II, (8), p. 90.

5. À propos de l'enfant défini comme un petit homme parleur, voir Confessions (texte établi et traduit par Pierre de Labriolle, $7^{\theta}$ éd. revue et corrigée, Paris : Société d'édition "Les Belles Lettres", coll. "Des universités de France", 1956), tome I, livre I, chap. VIII, § 13, p. 12. 
d'altérité dans les Confessions à la lumière de la logique des pronoms personnels.

Selon saint Augustin, nous quittons l'infans pour entrer dans la "seconde enfance". Le philosophe suit l'étymologie : infans, qui non farer ${ }^{6}$. Désormais l'enfant parle. La langue présuppose la présence d'autrui. Par définition, parler, c'est s'entretenir avec quelqu'un. Le puer loquens s'adresse à un locuteur privilégié, sa mère ou sa nourrice. Que peut-on prédiquer de cette relation enfant-mère? Posons le prédicat dyadique $P e$ qui abrège "parle à" et Pé, "est parlé par". II formalise l'échange linguistique entre Je $(x)$ et Tu $(y)$ :

\section{1. $(\forall \xi)(\exists \psi) \operatorname{Pe}(x, y) \int P e ́(y, x)$}

Complétons cette première formule par une deuxième :

2. $(\forall \xi)(\exists \psi)\left(P e(x, y) \int P e ́(y, x)\right) \supset(x=y)$

S'il en était besoin, cette équation souligne cette solidarité fondamentale avec autrui. Autrement dit, l'altérité est une condition de l'échange verbal. L'égalité du Je et du Tu $(x=y)$ ne peut être confondue avec une identité. ${ }^{7}$ Elle désigne l'union, mieux la communion, dans la communication - communication fusionnelle du bébé avec sa mère. Prévaut ici, en général, la reconnaissance familiale, puis sociale.

Adviendra $x \neq y$, c'est-à-dire : la différenciation d'avec la mère ou son substitut passe parfois par le conflit, mais structure l'avènement du principe d'identité : $x=x$. L'égalité Je $=$ Je nécessite la reconnaissance personnelle et sociale. Introduisons dans le prédicat dyadique l'objet de "parler à" renvoyant, par exemple, à Elle et/ou Lui (z). Cette troisième variable propositionnelle correspond, en latin, au datif ou encore à l'ablatif, précédé de la préposition de, pour "parler de". Nous obtenons cette troisième formulation:

$$
\text { 3. }(\forall \xi)(\exists \psi, z)(P e(x, y) \& P e(y, z)) \supset P e(x, z)
$$

6. "Je n'étais déjà plus le bébé qui ne parle point, mais l'enfant doué de la parole". Voir Confessions, op. cit., livre I, chap. III, § 8, p. 11-12.

Remarque. - Infans, qui désignait en latin classique l'enfant en bas âge, littéralement "qui ne parle pas", a pris en outre à basse époque les sens de puer, puella, "garçon, fille de six à quinze ans environ" et de liberi "enfants par rapport aux parents". Voir Oscar Bloch et Walther von Wartburg, Dictionnaire étymologique de la langue française, Paris : Éditions PUF, 1932, $7^{e}$ éd. 1986, p. 223.

7. Stella Baruk, article "Égalité», Dictionnaire de mathématiques élémentaires, Paris : Éditions du Seuil, 1992; nouvelle édition enrichie de trois index, Paris : Éd. du Seuil, coll. "Science ouverte", 1995, p. 395-402. 
L'abandon du Je infantile se fait sous l'influence de l'éducation. Cet agent moral réconcilie le Moi avec le Non-Moi (l'autre générique), plus concrètement l'autre plus jeune ou de même âge ou encore adulte. Cette reconnaissance réciproque définit la conscience sociale. L'éthicité s'exprimera à travers le Nous des contemporains. Parallèlement, le sujet découvre le véritable amour. II abandonne la relation chosale Je-Cela, au profit d'une relation plus riche car ouverte : la relation à autrui (Je-Tu) qui, tout le long de la vie, invente divers jeux.

\section{Le concept d'identité}

I < a (Idéal plus petit que l'objet a, objet de la jouissance : prévalence de la jouissance sur l'Idéal)

Dans un second temps, après la logique symbolique, découvrons la logique analogique entre l'observation du jeune enfant - dans le cadre d'une psychologie concrète "anthropologisante" - et le discours analytique. Saint Augustin part de l'expérience de satisfaction du nourrisson. II s'agit, suivant l'étymologie, de l'expérience de l'«assez" (satis, en latin). Tout son être est assujetti aux exigences du besoin et des appétits, en particulier organiques - boire, manger, dormir et excréter. L'expérience de la survie du versant organique du Je pourrait se formuler ainsi : le Je infantile, étayé sur ses besoins, son désir et son plaisir, s'exprime par son jeu avec sa mère ou son substitut. L'expérience de la satisfaction définit l'attachement objectal égoïste. La satisfaction immédiate de ces besoins exige l'allégeance de la mère. En effet, la loi des échanges implique l'amour de soi et l'agressivité à l'égard de l'autre. C'est la distance qui sépare le Je du Tu, en l'occurrence la personne la plus proche du bébé, la mère nourricière ou la nourrice, protectrice et parfois souffre-douleur. Le jeu complice, tendre ou tyrannique avec la mère ou son substitut apaise l'état de tension et structure le Je infantile (cause finale).

L'Esquisse d'une psychologie scientifique compléterait, pour une part, les propos de saint Augustin :

Cette sorte d'intervention exige que se produise une certaine modification à l'extérieur (par exemple apport de nourriture, proximité de l'objet sexuel), une modification qui, en tant qu' «action spécifique» ne peut s'effectuer que par des moyens déterminés. L'organisme humain, à ses stades précoces, est incapable de provoquer cette action spécifique qui ne peut être réalisée qu'avec une aide extérieure et au moment où l'attention d'une personne bien au courant se porte 
sur l'état de l'enfant. Ce dernier l'a alertée, du fait d'une décharge se produisant sur la voie des changements internes (par des cris de l'enfant, par exemple). La voie de décharge acquiert ainsi une fonction secondaire d'une extrême importance : celle de la compréhension mutuelle. L'impuissance originelle de l'être humain devient ainsi la source première de tous les motifs moraux. ${ }^{8}$

Saint Augustin joint aux observations empiriques des considérations autobiographiques. L'évêque d'Hippone ne peut être soupçonné d'angélisme à l'égard de l'enfance : "Ainsi, ce qu'il y a d'innocent chez l'enfant, c'est la faiblesse de ses organes, mais son âme, non pas! ${ }^{\prime \prime}$ " Tous ses jeux ne sont pas innocents. Persuadé de la perversité foncière du cœur humain corrompu par le péché originel, ${ }^{10}$ Augustin décrit l'éveil des inclinations mauvaises jusque dans le cœur de l'infans: petites colères effrénées, emportements, chantage rageur des larmes, pour asservir les grandes personnes à l'entour à ses caprices, c'est-à-dire les gens de condition indépendante et libre, ses propres parents et autres personnes d'âge mûr. Ces jeux tyranniques s'accompagnent d'une envie de "frapper" tous ceux qui ne se prêtent pas à son désir, "en tâchant, précise-t-il, de leur faire tout le mal possible, pour m'avoir refusé une obéissance ${ }^{11}$ ». La cause efficiente de la loi des échanges est une passion de l'âme. Augustin choisit l'exemple de la jalousie ordinaire liée au Je physiologique.

Un petit enfant "regardait fixement, pâle et amer, son frère de lait» (ibidem). II s'agit de l'épreuve du ressentiment face à un rival qui jouit de l'objet convoité - téter le sein maternel. Deux affects se manifestent chez

8. Sigmund Freud, Esquisse d'une psychologie scientifique, dans La Naissance de la psychanalyse, lettres à Wilhelm Fließ, notes et plans (1887-1902) publiés par Marie Bonaparte, Anna Freud, Ernst Kris, traduit de l'allemand par Anne Berman, Paris : Éditions PUF, coll. "Bibliothèque de psychanalyse", $1956 ; 5^{e}$ éd. 1986, $1^{\text {re }}$ partie, [11] : "L'épreuve de la satisfaction", p. 336.

9. "Ita imbecillitas membrorum infantilium innocens est, non animus infantium" (Confessions, op. cit., livre I, chap. VII, § 11, p. 10).

10 Est-il besoin de rappeler que les Évangiles dénoncent le péché jusque dans les racines secrètes du comportement (Matthieu, V, 27-37, VI, 12 sqq., XV, 1-20; Marc, VII, 14-23; Épitre aux Romains, V, 12-15)? Voir Hugues Derycke, "Le vol des poires, parabole du péché originel", Bulletin de Littérature ecclésiastique, Institut catholique de Toulouse, tome LXXXVIII, $n^{\circ} 3-4$, juillet-décembre 1987, p. 337-348.

Remarque. - Dans cette description de la jalousie infantile, saint Augustin a le souci de montrer qu'on ne peut pas le suspecter de manichéisme. L'homme est intrinsèquement pervers, semble-t-il suggérer, par crainte de ceux qui l'accusent d'être encore manichéen.

11. Confessions, op. cit., livre I, chap. VII, $\S 11$, p. 10. 
l'enfant jaloux : le blêmissement 12 et l'expression d'amertume (signifiants préverbaux). II blêmit d'une rage impuissante à s'approprier l'objet de son désir. Cette réaction affective résulte d'un processus primaire : l'expression faciale produite par le libre écoulement de l'énergie. Dans cet exemple, le Je volontaire et désirant fait l'expérience de la frustration et du manque. La jalousie n'est qu'un désir de possession, une volonté quelquefois acharnée de propriété exclusive.

Pour la théologie et la morale religieuse, la violence aveugle est l'expression du mal. Augustin s'interroge sur la corruption originelle de l'enfant. Autrement dit, il porte son attention sur le sens de ses jeux parfois cruels. Loin de la bonté naturelle et antérieure aux vices imputés à la société, l'enfance n'échappe pas aux conséquences du péché originel : «Mais si "j'ai été conçu dans l'iniquité", si "c'est dans le péché que ma mère m'a nourri dans son sein", où donc, je vous prie, ô mon Dieu, où ai-je été, Seigneur, moi, votre serviteur, où et quand ai-je été innocent? ${ }^{13}$ ".

Sur le plan psychologique, cette expérience formatrice et structurante limite de facto les prétentions du sujet à plier le monde à sa volonté. "Si on laissait faire à l'enfant ce qui lui plaît, il n'est pas de crime où on ne le verrait pas se précipiter", écrit saint Augustin. En l'absence du renoncement pulsionnel, les folies de l'enfance sont l'enfance de nos folies.

Des réflexions du père de la psychanalyse confirment les observations psychologiques de saint Augustin qui portent sur le développement des états de l'âme. L'autoanalyse de Sigmund Freud met au jour ses réactions de jalousie, aux alentours d'un an, à la naissance de son premier frère Julius. La naissance d'un enfant plus jeune bouleverse les relations familiales. II découvre que sa libido s'est tournée, entre deux ans et deux ans et demi, vers matrem, et que le nouveau-né est un intrus qui va capter

12. Confessions, traduction par Louis de Mondadon (s.j.), Paris, Éditions Pierre Horay et Cie, 1947, coll. "Le Livre de poche", n 2304, 1967, p. 27.

Jacques Lacan consigne dans son rapport : "Ainsi nove-t-il impérissablement, avec l'étape infans (d'avant la parole) du premier âge, la situation d'absorption spectaculaire : "il contemplait", la réaction émotionnelle : "tout pâle", et cette réactivation des images de la frustration primordiale : "et d'un regard empoisonné", qui sont les coordonnées psychiques et somatiques de iagressivité originelle». Voir "L'agressivité en psychanalyse» (1948), Écrits, Paris : Éditions du Seuil, coll. "Le champ freudien", 1966, p. 114-115. Ailleurs, J. Lacan avait reconnu : "[...] cette jalousie dont saint Augustin entrevoyait déjà de façon fulgurante la valeur initiatrice [...]" (“Propos sur la causalité psychique», 1946, Écrits, op. cit., p. 181).

13. Confessions, op. cit., livre I, chap. VII, § 12, p. 11. 
l'attention et l'amour de leur mère. Chez le très jeune Sigmund Freud "de méchants souhaits et une véritable jalousie enfantine ${ }^{14}$ " sont suscités par ce rival. La naissance d'un autre frère réanime la jalousie éprouvée à l'égard du premier. Sa mort, survenue quelques mois plus tard, laissera chez lui le germe d'un remords dont son autoanalyse porte les traces. De même, Sigmund s'allie à John, un fils d'Emmanuel (un des demi-frères de $S$. Freud) pour traiter avec violence et cruauté Pauline, sa nièce plus jeune d'un an. Elle est la victime de leur complicité dans les jeux précoces de la jalousie.

Qu'est-ce qui est en jeu, sinon la formation et le développement du Moi? Comme Sigmund Freud, sans doute, l'auteur des Confessions répondrait : le système perception-conscience dont les attributs sont la permanence, l'identité et la substantialité. Ici le centre de gravité est le Je égoïste uniquement capable d'un amour captatif qui cherche à posséder l'objet ${ }^{15}$.

Passons à la seconde enfance. Appelé pupus (petit garçon) ou pupa (petite fille), l'enfant est, par nature, un être qui joue, du hochet à l'attelage d'animaux, du cerceau à la toupie, etc. Le jeu est pour le Je enfantin son essence. Pour Roger Caillois, l'univers du jeu se distribue entre deux extrêmes : paidia et ludus ${ }^{16}$, la personnalité de l'enfant se forme en passant

14. Voir lettre LXX à Wilhelm Fließ (Vienne, le 3 octobre 1897), dans La naissance de la psychanalyse, op. cit., p. 194.

15. "Le moi, point virtuel du regard, où se reflètent les identifications, se voit dans le miroir de ce petit autre fantasmatique, cet objet petit autre. Auquel le chat enfant donne ses coups de griffe, au miroir. Tandis que l'enfant saint Augustin l'a perçu dévorant le sein de sa mère". Voir Jean-Pierre Faye, "Actes du temps qui parle. Le continent lacanoaméricain", Passages, octobre-novembre 1997, $n^{\circ} 86$, p. 11.

16. "À une extrémité règne, presque sans partage, un principe commun de divertissement, de turbulence, d'improvisation libre et d'épanouissement insouciant, par où se manifeste une certaine fantaisie incontrôlée qu'on peut désigner sous le nom de paidia. À l'extrémité opposée, cette exubérance espiègle et primesautière est presque entièrement absorbée, en tout cas disciplinée, par une tendance complémentaire, inverse à quelques égards, mais non à tous, de sa nature anarchique et capricieuse : un besoin croissant de la plier à des conventions arbitraires, impératives et à dessein gênantes, de la contrarier toujours davantage en dressant devant elle des chicanes sans cesse plus embarrassantes, afin de lui rendre plus malaisé de parvenir au résultat désiré. Celui-ci demeure parfaitement inutile, quoiqu'il exige une somme constamment accrue d'efforts, de patience, d'adresse ou d'ingéniosité. Je nomme ludus cette seconde composante" (Roger Caillois, Les jeux et les hommes. Le masque et le vertige, Paris : Éditions Gallimard, $1^{\text {re }}$ éd. 1958, édition revue et augmentée, coll. "Folio. Essais", 1967, p. 48). Voir également "Répartition des jeux" en annexe. 
du premier au second ordre de jeux. À sept ans, l'enfant quitte l'univers "paidiatique" pour celui de l'école.

Quelle est la cause de la paresse scolaire du jeune Augustin? "J'adorais le jeu» 17 , confie-t-il adulte. II préférait le jeu de paume aux leçons. Son amour du jeu (amor ludendi) est lié à l'orgueil - motif de tout péché : “(...) j'aimais dans les luttes l'orgueil de la victoire; les récits fictifs, qui, chatouillant mon oreille, y allumaient une plus avide flamme; une curiosité chaque jour plus forte pétillait dans mes yeux, et m'entrainait aux spectacles, divertissements des grandes personnes ${ }^{18}$ ".

Le Je en devenir passe par des phases; celle de l'apprentissage des arts et des lettres est vécue douloureusement par Augustin écolier qui ne comprend pas l'attitude des adultes. Le rapport éducatif contribue ici au malheur de l'enfant. Nous saisissons mieux son investissement réactif du jeu (principe de plaisir) lorsque nous lisons ce souvenir datant de son époque de formation : «(...) on me mit à l'école pour apprendre mes lettres; pauvre que j'étais, je ne voyais pas à quoi cela servait, et pourtant quand je me montrais paresseux à apprendre, je recevais des coups. Les grandes personnes trouvaient cela parfait ${ }^{19}$ ".

Ailleurs, le même souvenir lié au "dépit rageur", voire à l'«effroi" toujours présent et intense, lors de sa rencontre avec le principe de réalité, 20 toujours douloureux et blessant, le conduit à un réquisitoire contre l'école, en particulier contre ces éducateurs qui usent et abusent des châtiments corporels. "Les chevalets, les ongles de fer, et ces autres instruments de torture" exposent la violence des maîtres et des précepteurs. Ce décor anticiperait presque le boudoir sadien. Suite à sa vive indignation dans sa dénonciation des brutalités, on peut sans peine deviner les jeux (?) sadiques infligés aux récalcitrants.

Les effets 21 traumatisants de la violence demeurent ineffaçables. La psychanalyse nous apprend que plus l'apprentissage est réussi, plus facilement le souvenir de cette violence s'effacera de la conscience : c'est ici la preuve a contrario d'un apprentissage difficilement surmonté. Néanmoins les menaces et les représailles des maîtres furent intégrées au psychisme.

17. Confessions, op. cit., livre I, chap. IX, § 15, p. 14.

18. Idem, livre I, chap. X, §16, p. 15.

19. Idem, livre I, chap. IX, §14, p. 13.

20. Pour S. Freud, principe de réalité et principe de plaisir sont dans un rapport dialectique.

21. Chacun sait que les mots "accusation" et "causalité" ont la même étymologie. 
Elles accèdent, par l'objectivation de l'écriture, à la représentation ou contenu de pensée.

À décharge dans la critique de l'institution scolaire, le chapitre XII justifie la sévérité de l'éducation au nom de "l'ordre immuable" de Dieu selon lequel "toute âme déréglée trouve sa peine dans ses propres dérèglements 22 ", en l'occurrence ceux de l'amour excessif des jeux enfant et préadolescent. Parmi ses aversions, voire ses tourments, Augustin recense en tête la langue grecque, suivie de la lecture, de l'écriture et du calcul. Cependant d'autres disciplines trouvent grâce à ses yeux, à commencer par le latin .23 Par-delà l'appel implicite à humaniser les humanités, l'auteur conclut sur l'alternative entre être ou ne pas être - insondable décision de l'être et enjeu de la causalité du sujet face aux humiliations, aux souffrances : "Et l'étude même qui leur [aux écoliers] est imposée par la menace des peines, est une peine si vive, que souvent ils préfèrent à l'étude, les peines qui les obligent à l'étude. Qui ne frémirait, qui ne choisirait de mourir, si l'alternative était proposée de souffrir la mort ou de recommencer l'enfance? ${ }^{24}$ ".

L'alternative existentielle est une disjonction exclusive. Si le Je augustinien pouvait choisir son existence, il opterait pour la mort face à la vie maltraitée, en mémoire des redoutables punitions. Thanatos gagne, Éros est hors jeu. Ce choix a sans doute sa cause dans l'amour de la vie : vaut-elle la peine d'être vécue dans la violence? Ce choix négatif et violent (on ne peut transiger avec la loi du tiers exclu) est à la mesure de sa déception d'enfant face au comportement des adultes, éducateurs et professeurs.

Dans la vie quotidienne, on assure son salut par l'action propre à l'être désirant en se projetant toujours dans l'avenir. Présentement, l'acte d'assomption subjective passe par l'intégration de la souffrance et son dépassement par la sublimation. ${ }^{25}$ Comment devient-on sujet? Par l'acte

22. Les Confessions, éd. par A. Derrez (1835), Paris : Éditions Sand, coll. "Sagesse et spiritualité", 1997, livre I, chap. XII, p. 57.

23. Confessions, op. cit., livre I, chap. XIII, $\$ 20$, p. 17.

24. La Cité de Dieu (De Ciuitate Dei), traduction du latin par Louis Moreau (1846), revue par Jean-Claude Eslin, Paris : Éditions du Seuil, coll. "Points Sagesses", 1994, vol. III, livre XXI, chap. XIV, p. 251.

25. "Mais cette même incapacité de la pulsion sexuelle à procurer la satisfaction complète, dès qu'elle est soumise aux premières exigences de la civilisation, devient la source deș œuvres culturelles les plus grandioses, qui sont accomplies par une sublimation toujours plus poussée de ses composantes pulsionnellos", rappelle $S$. Freud dans la deuxième Contribution : "Sur le plus général des rabaissements de la vie amoureuse" (1912), traduit de l'allemand par Jean 
de construction et de destruction : la dénonciation d'Augustin et l'énoncé de son jugement existentiel lui permettent d'affirmer son Je hors des jeux cruels de ses persécuteurs. Le Je s'affirme en refusant la loi commune du monde. Par conséquent, il devient un sujet par sa décision d'être. ${ }^{26}$

Advient l'adolescence avec son enthousiasme juvénile et ses passions fiévreuses qu'un psychanalyste contemporain nomme joliment "le temps de la promesse pubertaire27". Elle correspond également à une crise d'identité et à l'errance. Saint Augustin s'interroge sur ce Moi étranger à lui-même qui dit Je. L'essence d'un être, déclare Aristote, c'est sa fin. Or dans les miasmes de l'adolescence, aux prises avec les passions et les appétits, quelle fin ont les jeux amoureux et érotiques pour le Je? Use-t-il de sa raison adolescente?

Avant sa conversion, Augustin adolescent est un représentant de l'humanité moyenne. Les Confessions peignent une nouvelle étape du Moi aux couleurs de la passion. À la puberté, l'irruption du sexuel se fait dans l'intensité qui ignore les scrupules ${ }^{28}$ et la claudication de l'âme. La faim d'excitations et le déchaînement des forces - celles des pulsions sexuelles - submergent le sujet. L'adolescent découvre l'état amoureux, c'est-à-dire la vie libidinale. Durant l'enfance, le désir s'étaye sur les fonctions vitales (corps organique). À l'adolescence, la libido réactivée trouve une nouvelle vigueur dans les plaisirs et les jeux sexuels ou non. Unis dans la pulsion, corps et psychisme explorent tous les modes de jouissance. Entre besoin et désir, cet appel aux sens par ce dernier dynamise la quête de l'autre dans l'amour.

Laplanche, chap. IV : "Contributions à la psychologie de la vie amoureuse", dans La Vie sexuelle, Paris : Éditions PUF, coll. "Bibliothèque de psychanalyse", $1^{\text {re }}$ éd. $1969,11^{\theta}$ éd. 1997, p. 65. Voir également Denis de Rougemont, L'Amour et l'Occident, édition définitive, Paris : Librairie Plon, 1972; rééd. Paris : Éd. “10/18", n 34, 1979, réimpression 1995, livre II, chap. III : “Agapè ou l'amour chrétien", p. 68-71.

26. Dans la tradition philosophique, le désir est une modalité de l'imagination qui comble un manque. Chez l'être parlant, le désir n'est pas promis à la jouissance, du moins toute jouissance ne peut être que substitutive. À l'arrière-plan de la structuration de la personnalité d'Augustin se tient la figure maternelle Monique - qui nourrit en lui certaines considérations philosophiques, enrichies de remarques psychologiques, en particulier sur les interdits inconsciemment intégrés (introjection et constitution partielle du Surmoi). Sur le nœud de sentiments liant Augustin à sa mère dominatrice, voir Jacques Chomarat, art. cité.

27. Jean-Jacques Rassial, L'Adolescent et le Psychanalyste, Paris : Éditions Rivages, coll. "Psychanalyse", 1990, p. 11.

28. Au sens premier, scrupulus signifie en latin "petite pierre pointue». 
Dans le deuxième livre, nous mesurons la distance qui sépare l'amour charnel de l'amour oblatif ou amour de communion. L'adolescent demeure prisonnier de son Je et de ses jeux égoïstes qui assouvissent la satisfaction narcissique, d'où son désir de découvertes, d'expérimentations, son avidité. Le professeur de rhétorique ne ménage pas ses effets stylistiques. La profusion de tropes et leur redondance exprime l'excès de l'univers physique et psychique de l'adolescence, âge des "ronces des passions» : “(...) j'étais en pleine effervescence, je m'abandonnais à l'impétuosité du torrent intérieur qui m'entraînait loin de vous [Dieu], et je transgressais tous vos commandements $(. . .)^{29}$ ".

Toutefois, "prétendre que la chair est cause de l'immoralité et de tout vice quel qu'il soit, que l'âme vivant ainsi n'obéit qu'aux impulsions de la chair, c'est ne pas méditer sérieusement sur toute la nature de l'homme ${ }^{30}$ ", rappelle saint Augustin à tous ceux qui seraient tentés de confondre péché et chair. Ce n'est pas par le mépris du corps que lâme assure son salut. La misère de l'humaine condition (souillures et délits) vient du mal. La liberté débridée fait l'humanité pécheresse.

Être pubère ${ }^{31}$ ou nubile n'empêche point les jeux et les facéties dans les rues dont les passants font les frais. L'adolescent, ce quasi-adulte (?), a parfois une conduite immature. Le Je de l'âge d'homme passe par des expériences ludiques, en particulier celle qui a marqué Augustin dans sa "seizième année de l'âge de ma chair". II précise les circonstances et explique le motif du pillage d'un poirier:

\footnotetext{
Dans le voisinage de notre vigne se dressait un poirier chargé de fruits qui n'avaient aucun attrait de saveur ni de beauté. Nous allâmes, en une bande de jeunes vauriens, secouer et dépouiller cet arbre, vers le milieu de la nuit (nous avions prolongé nos jeux sur les places jusqu'à cette heure, selon notre détestable habitude). Nous en rapportâmes de grandes charges, non pour en faire régal, mais simplement pour les jeter aux pourceaux. Si nous en mangeâmes un peu, tout notre plaisir vint de ce que cela nous était défendu. 32
}

29. Confessions, op. cit., trad. par P. de Labriolle, livre II, chap. II, § 4, p. 32.

30. La Cité de Dieu, op. cit., vol. II, livre XIV, chap. III, p. 148.

31. La manifestation de la virilité au bain public fait accéder Augustin à la puberté physique et sociale (Confessions, op. cit., livre II, chap. III, § 6, p. 33). À l'âge où l'adolescent devient homme, le père a le pouvoir de donner à son fils la toge virile.

32. Confessions, op. cit., livre II, chap. IV, § 9, p. 36. 
Qu'est-ce qui (é)meut le Je dans l'objet dérobé, sans être poussé par le besoin? L'auteur des Confessions s'en explique : "Ce n'est pas de la chose convoitée par mon larcin, mais du larcin même et du péché que je voulais jouir33". Ce vol ludique sans objet, ni consommation véritable ni vente, le satisfait. II a pour but de convertir le désir en excitation, engendrée par la transgression qui cimente le groupe. Protégés par l'obscurité, Augustin et ses complices se cachent du regard d'autrui... Mais non de Dieu.

Rapportons cette infraction ou ce délit (?) à l'espace symbolique. D'après la Bible, la bassesse correspond au péché. ${ }^{34}$ Sous l'angle théologique, le vol des poires est la parabole du péché originel..$^{35}$ Quant à l'acte pulsionnel, le vol met en jeu des forces qui plongent dans le tréfonds du psychisme. ${ }^{36}$ La dernière phrase de l'extrait livre le sens du larcin. On peut comprendre cet acte, apparemment gratuit, dans le rapport du sujet à l'ordre symbolique. L'acte de voler est pris dans cet ordre indépendant de l'objet dérobé. S'emparer des poires pour s'en débarrasser réalise une autre fin. La complicité ludique dans la transgression vise à enfreindre, plus que les interdits parentaux, voire ceux de la société, un commandement de Dieu (Exode, XX, 15). N'est-ce pas le défi et l'affirmation du Moi adolescent? Infractions et délits vécus comme des jeux, crimes dans certains cas, assoient l'identité. En termes analytiques, c'est la manifestation du désir de l'adolescent en tant que sujet. D'autre part, l'acte délinquant crée d'autres

33. Ibidem. Saint Augustin montre que le vice est toujours une contrefaçon de Dieu (livre II, chap. VI, § 13).

34. Satan, ange déchu, est l'instigateur de la chute d'Adam et Ève (Genèse, III, 1 7, 13-15; Deuxième Épître aux Corinthiens, XI, 3; etc.). Outre l'évitement du péché, le chrétien a pour devoir de s'élever (l'ascension intérieure de l'expécheur) ainsi que le recommande, par exemple, l'Épitre aux Colossiens (III, 1 2). La conversion, et plus encore l'extase, est une remontée : “C'est vers cet asile que l'amour nous soulève, et que "votre Esprit-Saint exalte notre bassesse, arrachée aux portes de la mort". La paix est pour nous dans la "bonne volonté". Tout corps tend, en vertu de sa pesanteur, vers la place qui lui est propre : mais un poids ne tend pas nécessairement vers le bas : il tend vers la place qui lui est propre" (Confessions, op. cit., tome II, livre XIII, chap. IX, $\S 10$, p. 372-373).

35. Pour le commentaire théologique de cet épisode, voir Jean-Marie Le Blond, Les Conversions de saint Augustin, op. cit., p. 68-71, et l'article de Hugues Derycke (v. note 10).

36. Ceci correspond à la définition topique du Moi psychanalytique, ou métapsychologique, qui a "la tête en bas, comme l'homoncule des anatomistes". Voir Sigmund Freud, Le Moi et le Ça (1923), chap. V : "Les relations de dépendance du Moi", Essais de psychanalyse, traduit de l'allemand par Jean Laplanche, Paris : Éditions Payot, coll. «Prismes», $n^{\circ} 7,1987$, p. 263, note 1. 
règles reconnues par le groupe qualifié de "vauriens", et un autre lieu dont la valeur n'existe que pour eux. Chacun d'eux constitue deux repères d'identification. Autrement dit, le jeune Augustin passe de la reconnaissance du père à celle de ses pairs.

La crise d'identité se déroule durant cette construction du Moi. Or le mot crise signifie en grec "décision" : décision d'être, avec ses provocations et ses excès. L'acte délictueux d'Augustin, ce "crime nocturne" comme il l'appelle dramatiquement, peut être compris comme une compensation à la soumission de son enfance, en particulier à l'école. II prend donc place parmi les jeux compensatoires.

\section{I > a (Idéal plus grand que l'objet a)}

Du vol à l'envol : cette formule lapidaire désigne le dépassement de cet âge critique en quête de soi. La satisfaction passagère de l'excitation dans la transgression, la promiscuité et le divertissement négligent finalement l'être authentique. Augustin adolescent est accablé par le mal et vit l'éclipse de l'Absolu. Des tendances le dominent; elles emprisonnent le Je qui se croit libre. Les excès et les débordements sont des reliquats infantiles qui se modifient au cours de l'éducation avant d'atteindre la maturité. ${ }^{37}$ L'expérience de la vie, ou principe de réalité, fait régresser progressivement les jeux amoureux, sociaux, intellectuels... immatures. C'est la formation réactionnelle de la personnalité.

La violence contribue à la constitution de notre identité. Écolier, c'était l'apprentissage des règles que l'autre impose. L'adolescent en quête d'identité les défie. Pour parvenir à la conscience de soi et à la maturité, le sujet doit intégrer et surmonter le jeu des forces libidinales. En d'autres termes, la pérennité des jeux adolescents menace la réalisation des dispositions sous-jacentes, celles de la méditation et de la réflexion qui répondent aux interrogations et indiquent le sens (contenu et direction). Car tout dans la vie n'est pas jeu. À l'âge adulte, s'affirme en effet la prééminence de la conscience et de la lucidité. II s'agit d'une prise de conscience et de

37. Sur la transformation des affects, voir Sigmund Freud, L'Interprétation des rêves, trad. par I. Meyerson, nouvelle édition augmentée et entièrement révisée par Denise Berger, Paris : Éditions PUF, 1926 \& 1967, p. 513-515. Voir également la conclusion de sa xxxiie conférence : "Angoisse et vie pulsionnelle", Nouvelles conférences d'introduction à la psychanalyse (1933), traduit par Rose-Marie Zeitlin, Paris, Éditions Gallimard, coll. "Connaissance de I'Inconscient", série : "CEuvres de Sigmund Freud (traductions nouvelles)", 1984. 
confiance du Moi. La raison théorique et pratique n'est-elle pas un facteur déterminant d'intégration sociale, d'ouverture à l'autre et la garantie de notre identité? L'idéal religieux, mais aussi moral et éducatif (la transmission d'idéaux), contribue également à se défaire de la perversité de notre volonté, de l'égoïsme sans limites. Aux désirs insatiables et à leurs composantes pulsionnelles - l'incoercible besoin d'amour charnel répond la maîtrise de soi prêchée par saint Augustin. Elle exclut tout amour tumultueux, tout jeu sexuel. Elle encourage même l'abstinence. Augustin défend donc le mariage qui vise la procréation : le désir est canalisé et fixé par la loi des hommes et de Dieu. Pour la cause du royaume des cieux, il préférerait devenir eunuque plutôt que luxurieux (Matthieu, XIX, 12). ${ }^{38}$

La sagesse, avons-nous dit, se caractérise par l'ouverture intellectuelle (la connaissance intuitive et réflexive de l'âme), ${ }^{39}$ et l'ouverture à l'autre le prochain ou l'ami(e). Cette opération passe par l'abandon de l'égocentrisme et des jeux puérils de l'enfance. La vérité du sujet s'exprime dans l'acte. La prépsychologie d'Augustin, ou description des étapes formatrices de l'âme, associe l'anthropologie chrétienne à une anthropologie existentielle. A contrario, le théâtre des jeux dérisoires de l'adolescent ou de l'adulte immature nous rappelle, si besoin était, notre fragile humanité en perte ou, pis, en absence de transcendance.

Le titre du livre XI des Confessions, "Élévations sur les mystères", désigne une manière de réfléchir sur ce que la dogmatique chrétienne appelle le mystère. Le temps appartient au même ordre de pensée que les dogmes chrétiens. La lecture de la Genèse encourage saint Augustin à s'interroger sur le temps, celui du premier kairos, la Création.

L'âge adulte correspond à la conscience de soi qui accède enfin aux interrogations essentielles. Le Je ludique fait place au Je philosophique ou cogito. Le temps nous est "familier" et "connu», il est réel pour la conscience. II s'agit de l'expérience muette, c'est-à-dire non conceptualisée, du temps. Augustin s'emploie à le théoriser en répondant, tout d'abord, à la question : quel est le mode d'être du temps, autrement dit quelle est sa réalité ontologique? Tout le monde connaît sa réponse. Le temps appartient à l'essence même de la conscience de tout homme venant au monde. Son élucidation renvoie à la question du statut ontologique du présent, celui qui correspond au fondement de l'être. Les

38. Confessions, op. cit., tome I, livre II, chap. II, § 2, p. 30-31.

39. Voir J.-M. Lamarre, Saint Augustin, La Trinité, Paris : Éditions Louis Magnard, coll. "Texte et contextes", 1985, p. 56-118. 
parties du temps sont toutes vécues par la conscience de soi (la subjectivité) au présent en tant qu'il passe. Par conséquent le temps est et n'est pas à l'image de notre être composé d'être et de néant. La conscience augustinienne ne fait que se situer dans l'ordre du temps. Être homme signifie être éclairé par la raison et la conscience. II est au présent une anticipation subjective.

"Et quoi, qui es-tu ?" J'ai répondu : "Un homme"." Nous avons mis en exergue cette interrogation aphoristique de saint Augustin, car l'auteur des Confessions s'attache aux moments cruciaux de l'enfance, de l'adolescence et de l'âge adulte. II s'emploie à décrire l'être en devenir. Être homme, c'est refuser cet abandon sans réserve à la satisfaction immédiate. Au point de vue religieux, l'ldéal de saint Augustin pourrait s'élever à la hauteur d'un concept : I'homme augustinien - bien que la sainteté ait, pour la plupart d'entre nous, la figure de l'impossible. Du moins, la libération de l'âme asservie par les «liens du désir sensuel, (...) et la servitude des affaires temporelles ${ }^{40}$ » demeure possible.

Signalons qu'au livre huitième des Confessions, des présages ou mieux des signes ${ }^{41}$ précèdent la conversion d'Augustin. Retenons deux éléments d'apparence insignifiante qui nous semblent dignes d'attention parce qu'ils annoncent une rupture radicale entre le Je ludique et celui qui adhérera prochainement au christianisme :

- Ponticianus est l'hôte d'Augustin et d'Alypius. Notons la simplicité de la scène et du décor : "Nous nous assîmes pour causer. Le hasard voulut que, sur une table de jeu, devant nous, il (Ponticianus) remarquât un livre, le prît, l'ouvrît, et y trouvât les Épîtres de l'apôtre Paul42».

L'ablatif "supra mensam lusoriam" n'est pas anodin. La table de jeu destinée au ludus appartient à la catégorie agôn, au sens de Roger Caillois. ${ }^{43}$ Elle perd ici sa valeur d'usage. Nous rompons avec les jeux triviaux des loisirs en famille et entre ami(e)s, pis encore avec la passion du jeu. La table de jeu ouvre céans au sacré, à la divinité du Verbe, plus

40. Confessions, op. cit., tome I, livre VIII, chap. VI, §13, p. 186.

41. "Dieu, qui parle aux hommes à travers sa création, leur fait aussi signe de manière spéciale par des actions qui suscitent l'étonnement». Voir Xavier Léon-Dufour, article "signe", $2^{e}$ définition, dans Dictionnaire du Nouveau Testament ( $2^{\ominus}$ éd. revue, Paris : Éditions du Seuil, coll. "Livre de vie", $n^{\circ} 131$, 1975, p. 496).

42. Confessions, op. cit., livre VIII, chap. VI, $\S 14$, p. 187. (Nous nous sommes permis de rétablir la concordance des temps dans la subordonnée.)

43. Voir Annexe. 
précisément aux Épîtres aux Romains de saint Paul. Cet événement est interprété comme un message de Dieu. II est source d'une crise intérieure dans laquelle Augustin exprime son désarroi 44.

- Quelques chapitres plus loin, un second signe révélateur a le même sens. Dans le jardin de Milan, un chant le bouleverse :

Et tout à coup j'entends une voix partie de la maison voisine, voix de garçon ou de jeune fille, je ne sais, qui chantait et répétait à diverses reprises : "Prends, lis ! Prends, lis !" Et aussitôt, changeant de visage, je cherchai très attentivement à me rappeler si c'était un refrain en usage dans quelque jeu d'enfant; et rien de tel ne me revint à la mémoire. 45

Ce signe de la providence est interprété comme "un ordre divin» l'enjoignant d'ouvrir le Nouveau Testament. Augustin tombe alors sur le verset de l'Épître aux Romains de saint Paul (XIII, 13) qui invite les fidèles à renoncer aux voluptés et à "revêtir Jésus-Christ». Cet événement vainc ses dernières réticences. Le refrain n'appartient pas aux comptines enfantines. Sinon il entrerait dans la catégorie alea (chance) située du côté de la paidia, toujours selon la classification de Roger Caillois. Nous sortons, comme précédemment, du jeu pour entrer dans la dimension religieuse et mystique.

Reste à comprendre le choix d'ouvrir au hasard la Bible pour en tirer conseils et enseignements. Une telle pratique s'apparente à la divination correspondant à la classe alea de R. Caillois. Ou, dans le second exemple, cet enfant qui, par sa voix mystérieuse, triomphe des ultimes résistances d'Augustin à sa conversion. Ne sont-ce pas encore des jeux, alors que nous parlons de deux ordres séparés, voire opposés (profane/religieux)? Augustin vit, à cet instant, la version chrétienne de pratiques divinatoires paiennes. L'explication du traducteur Pierre de Labriolle lève la contradiction en se débarrassant de la séduction du monde sensible et du désir de l'objet trivial :

Chose curieuse, dans l'Épitre LV, 37 (Patrol lat., XXXIII, 222), adressée à lanuarius un an ou deux après la publication des Confessions, Augustin désapprouvera la coutume de tirer au sort (sortes legere) dans les Évangiles : il est vrai que les consultations

44. Confessions, op. cit. livre VIII, chap. VII, § 16, p. 189-190.

45. Confessions, op. cit. livre VIII, chap. XII, § 29, p. 199-200. 
qu'il blâme portaient sur des intérêts purement matériels (negotia saecularia). 46

Ouvrir la Bible au hasard ne peut évidemment pas être rangé parmi les jeux triviaux. L'interpréter comme un sort semble autorisé si cette pratique vise à améliorer l'âme. Saint Augustin exerce la divination, du moins la tolère-t-il sous certaines conditions. Pourquoi en viendra-t-il à la condamner?

II s'oppose certainement à elle au nom de sa conception de l'homme (son éthique est indissociable de son ontologie). L'affirmation de soi par la pratique du hasard conduit à la passivité de son utilisateur : le jeu est puissance (virtù) négative. Autrement dit, les jeux du sort imposent un choix étranger à la volonté du joueur en réduisant sa liberté à l'impuissance. Cette interdiction relève d'une protestation ontologique : la maîtrise de soi implique la mort du jeu. Peut-on maîtriser un jeu de hasard? Le pouvoir octroyé à la contingence ne dénie-t-il pas le sérieux du monde et de l'existence? Cette raison a de quoi irriter saint Augustin.

De plus, le tirage au sort de la Bible offre un caractère irrationnel fût-il masqué par sa prétention à communiquer avec le sacré. Sa condamnation des jeux du sort (alea) se justifie également par un motif théologique : il ne supporterait plus que la parole de Dieu devienne, en quelque sorte, l'objet d'une loterie. Au jeu de clefs bibliques, il préfère l'étude et la méditation des Écritures, c'est-à-dire la sagesse philosophique

46. Ibidem, p. 200, note 1. L'Église ancienne confiait aux enfants l'ouverture des livres saints. Elle les interprétait comme un sort. Sur le hasard providentiel source de révélations, voir Auguste Bouché-Leclercq, Histoire de la divination dans l'Antiquité, Paris : Emest Leroux, éditeur, 1879-1882, 4 vol. in-8, tome IV, livre II, chap. II : "Les sorts", p. 145-159. Quant au rapport divination et christianisme, l'auteur précise : "Enfin la divination sort triomphante de ces interminables débats : le christianisme l'accepte, en répudiant comme démoniaque celle qui ne relève pas de lui. II n'en élimine que les rites extérieurs, comme entachés de magie, et leur substitue la prière; tandis qu'il conserve tout ce qui vient spontanément de Dieu, songes, visions, inspirations prophétiques. L'humanité n'aurait pas supporté encore une Providence muette : la prière n'est plus uniquement un monologue résigné auquel Dieu se serait interdit de répondre autrement que par des actes. II faut attendre que l'esprit religieux se soit affiné et déshabitué de cette surexcitation inquiète dont s'alimentait la ferveur des premiers siècles, pour trouver la révélation pratique resserrée dans deux formes purement subjectives, la sollicitation de la grâce, et, chez les âmes les plus parfaites, le colloque intérieur avec Dieu " (op. cit., 1879, tome I, p. 103).

Rappelons que saint Augustin regarde la divination paĩenne comme démoniaque, voir La Cité de Dieu, op. cit., vol. III, livre XVIII, chap. V (p. 17-18) et livre XXII, chap. VIII (p. 296-311). 
qui seule peut satisfaire une conscience religieuse. Voilà, selon nous, les raisons pour lesquelles Je ne joue plus - notre titre se trouve justifié.

Chez saint Augustin, "l'arbre existentialiste" s'enracine dans une ontologie qui n'a de sens que pour l'adulte capable de tourner son regard sur les étapes de son passé, c'est-à-dire de réaliser son examen de conscience (titre du livre X). Emmanuel Mounier affirme : "Le transcendant y est saisi directement comme plénitude commençante et gloire entrevue de l'être, dans l'expérience générale du dépassement ${ }^{47}$ ".

La transcendance intérieure peut emprunter cette voie heureuse. Celle-ci nécessite, en tant que condition nécessaire, une connaissance de notre être. La quête plus ou moins laborieuse de l'intériorité, c'est-à-dire la connaissance de notre Moi profond et authentique, réalise le "Connais-toi toi-même" socratique. Le jeu du Je correspond à la face noble de l'homme, celle de l'éveil de la conscience dans un rapport cruciforme : lien horizontal à l'autre et vertical à la transcendance. ${ }^{48}$ Cette dernière ne peut être confondue avec une quelconque extériorité, suivant la propension habituelle. La transcendance se situe et se déploie dans l'intériorité spirituelle: "Interior intimo meo", écrit saint Augustin, reprenant la célèbre formule évangélique, le royaume de Dieu est au-dedans de vous. Grammaticalement, la proposition «je me cherchais moi-même» exprime la dualité entre le sujet (je) et le complément d'objet ( $m e)$. Cette affirmation manifeste le travail de la conscience sur elle-même, c'est-à-dire l'activité réflexive ou conscience de soi.

Conformément aux Évangiles, l'union de l'âme à Dieu exige le dépouillement. Le Je, chez Augustin, renonce aux trois libido : libido sentiendi, libido sciendi et libido dominandi (désir de sensation, de connaissance et de domination) - lesquelles anticipent les trois ordres de Blaise Pascal : la chair, l'esprit et le cœur.

47. Emmanuel Mounier, Introduction aux existentialismes, op. cit., p. 182. L'auteur regroupe dans une démarche commune la réminiscence platonicienne, la mémoire de saint Augustin, le “ Tu ne me chercherais pas si tu ne m'avais pas trouvé " de Blaise Pascal, le "devant Dieu" de Søren Kierkegaard.

Remarque. - Sur le rapport entre l'introspection et la mémoire des sens, la mémoire intellectuelle et la mémoire des sentiments, voir Joël Candau, Anthropologie de la mémoire, Paris : Éd. P.U.F., coll. "Que sais-je?", n 3160, 1996, p. 24-27.

48. Être saint, n'est-ce pas se laisser aimer par Dieu et en tirer les conséquences? 
Un esprit ironique 49 reprocherait peut-être à l'Ego augustinien l'exclusion du jeu, au sens mécanique. Le Je en devenir n'aurait plus la possibilité de se mouvoir! Cette accusation relève de l'ignorance. Saint Augustin reconnaît le libre arbitre, c'est-à-dire l'espace de l'être. En d'autres termes, l'homme est responsable car il a en son pouvoir le choix ${ }^{50}$. Dans le Je mature, action, mobilité et réflexion constituent notre espace intérieur. Toutefois les passions ludiques - commander, voir, sentir - doivent à tout prix éviter l'orgueil. En effet, la superbia est dénoncée comme la cause du péché dont s'accuse souvent saint Augustin : "Le poids de mon orgueil me précipitait dans l'abîme ${ }^{51}$ ".

Le livre $X$ clôt la partie autobiographique. Celle-ci constitue un temps fort de son itinéraire. La découverte de soi permet à saint Augustin de réorienter son désir vers Dieu. En termes d'investissement, son choix d'objet d'amour est exclusif. Amusements puérils et futiles, jouissances immédiates et jeux plus ou moins vicieux de la vie sont exclus. Par suite, le Je augustinien ne joue ni avec le sérieux de l'existence, ni avec l'idée de Dieu et de ses attributs (De Trinitate), car Dieu est, selon la formule du père Marcel Neusch, la jouissance parfaite de l'objet désiré. 52 Cet amour échappe d'ailleurs à l'alternative jouissance ou désir.

Enfin, lorsque «lalangue» 53 joue sur le signifiant, la jouissance produit la diaphore jouis-sens. Le jeu de mots laisse entendre le contentement du $\mathrm{Je}^{54}$ en quête du sens de l'existence dont le futur père de l'Église est le héraut, à la fois messager et défenseur du christianisme.

49. Ironie, selon l'étymologie, «interrogation" qui procède inévitablement d'un déplacement de signification.

50. Voir saint Augustin, Contre le manichéen Félix [Contra Felicem manichaeum], II, IV.

51. Confessions, op. cit., livre IV, chap. XV, §27, p. 86 .

Remarque. - Les livres II et III décrivent les trois concupiscences, c'est-à-dire les trois formes réunies ou dissociées du péché : la libido ou tentation de la chair (livre II, chap. I, § 8), la superbia ou tentation de l'orgueil dont il est question ici, et la curiositas ou tentation des yeux, ceux du corps comme ceux de l'esprit (livre III).

52. Marcel Neusch (père), Initiation à saint Augustin. Un maître spirituel, Paris : Éditions du Cerf, coll. «Épiphanie. Biographies», 1996, p. 211.

53. "La batterie signifiante de lalangue ne fournit que le chiffre du sens. Chaque mot y prend selon le contexte une gamme énorme, disparate, de sens, sens dont l'hétéroclite s'atteste souvent au dictionnaire" (Jacques Lacan, Télévision, Paris : Éditions du Seuil, coll. «Le champ freudien», 1974, p. 21. L'homophonie qui suit se situe page 22).

54. Voir la joie existentielle, note 58. 


\section{Conclusion}

La lecture des Confessions vaut autant pour sa réflexion théólogique que ses descriptions et analyses psychologiques du genre humain à travers cette (en)quête sur l'existence. On ne peut qu'être sensible à la dimension relationnelle de l'être ainsi qu'à la formation de l'identité tout au long d'une vie. Depuis la naissance, l'altérité demeure au service de lidentité. De la puissance à l'acte, l'être tend à développer ses virtualités afin que le sujet advienne. L'identité engendre des jeux qui dépendent des besoins physiologiques, des tendances psychologiques et psychiques, de la volonté, des désirs conscients, voire inconscients. Les jeux du Je renvoient chacun à ses propres expériences. Celles-ci se placent sous la vigilance de la conscience morale ou instance surmoïque, 55 renforcée par la vigilance chrétienne pour les croyants. Saint Augustin découvre son identité sous le regard de Dieu. II libère son Je soumis au destin pulsionnel. En conséquence le double je(u) - vices privés, vertus publiques - est évidemment prohibé au risque de se mépriser et, pour une conscience religieuse, de commettre une trahison insondable des Écritures. Faut-il alors préciser qu'il faut dans la vie jouer franc je(u)?

Finalement les Confessions sont un modèle d'espérance. II n'est pas question d'envisager la vie bienheureuse comme la promesse à venir, mais comme une détermination présente de l'être humain. Désormais débarrassé des jeux blâmables, le sujet a affaire au "cœur». Le cor désigne, suivant notre perspective, l'ancrage du Je au cœur de l'être. II assume, en effet, une fonction existentielle (support de l'identité), morale (source des sentiments nobles) et théologique (voie de salut et voix de Dieu). ${ }^{56}$ Pour le théologien du Verbe, la prière dans l'intime du cœur humain se fait supplique, invocation ou louange. Le discours orant de l'angoisse, de la quête et du désir de lâme assure le dialogue avec Dieu : le Je augustinien se situe sur la voie du Créateur.

55. Voir La Gourmandise du Surmoi, dix-huitième journée d'étude du Centre de recherche de l'enfant dans le discours analytique, Toulouse : Presses universitaires du Mirail, coll. "Séries de la Découverte freudienne", vol. XVIII, janvier 1996.

56. Voir $M^{m e}$ Catherine Marès (née de Laurens de Saint-Martin), Recherches sur la notion de cor dans les Confessions de saint Augustin, D.E.S. dactylographié de lettres classiques, Université de Paris I, 1958-1959, en particulier $2^{\Theta}$ partie : "Augustin et ses sources : l'expression du cor selon différents "plans d'être" ", p. 42-72. Voir également Gérald Antoni, La Prière chez saint Augustin. D'une philosophie du langage à la théologie du Verbe, Paris : Librairie philosophique J. Vrin, coll. "Philologie \& Mercure. La tradition de l'humanisme latin", 1997, p. 9-11 passim. 
Philosophe de l'existence, saint Augustin nous invite à la vérité et à la plénitude du Moi - le Je est défini analytiquement comme le plus authentique sujet ${ }^{57}$ - autrement dit le sujet désirant atteindre au bonheur 58 Bien que cet idéal soit partagé par le commun des mortels, l'anthropologie augustinienne, comme la psychanalyse, parle inévitablement de la césure de l'être.

\author{
Christian Talin \\ Université de Paris I (Panthéon-Sorbonne)
}

57. Sigmund Freud, Nouvelles conférences d'introduction à la psychanalyse, op. cit., xxxie conférence : "La décomposition de la personnalité psychique", [64], p. 82.

58. Dans la perspective du bonheur existentiel, nous pensons à la description de Robert Misrahi : "Cette plénitude est à la fois la jouissance du désir et la réjouissance du désir comblé ; mais elle est aussi, en même temps, l'appréhension intuitive, c'est-à-dire interne et directe du sujet par lui-même comme signification et comme valeur. La conscience, dans et par l'acte de la joie substantielle, se saisit alors comme accord avec elle-même et se réjouit de sa propre "complétude"" (Le Bonheur. Essai sur la joie, Paris : Éditions Hatier, coll. "Optiques. Philosophie», 1994, p. 77). 


\section{Annexe}

Répartition des jeux

(Roger Caillois, Les jeux et les hommes, op. cit., p. 92.)

\begin{tabular}{|c|c|c|c|c|}
\hline & $\begin{array}{c}\text { Agôn } \\
\text { (compétition) }\end{array}$ & $\begin{array}{c}\text { Alea } \\
\text { (chance) }\end{array}$ & $\begin{array}{c}\text { Mimicry } \\
\text { (simulacre) }\end{array}$ & $\begin{array}{c}\text { llinx } \\
\text { (vertige) }\end{array}$ \\
\hline Paidia & [courses, luttes, etc.] & $\begin{array}{l}\text { comptines } \\
\text { pile ou face }\end{array}$ & $\begin{array}{l}\text { imitations enfantines } \\
\text { jeux d'illusions }\end{array}$ & $\begin{array}{l}\text { "toumis" } \\
\text { enfantin }\end{array}$ \\
\hline vacarme & non réglées & & poupées panoplies & \\
\hline agitation & athlétisme & & $\begin{array}{c}\text { masques } \\
\text { travesti }\end{array}$ & manège balançoire \\
\hline fou rire & boxe billard & & & $\begin{array}{l}\text { valse } \\
\text { volador }\end{array}$ \\
\hline cerf-volant & escrime dames & pari & théâtre & $\begin{array}{c}\text { attractions foraines } \\
\text { ski }\end{array}$ \\
\hline solitaire & & roulette & & \\
\hline réussites & football échecs & & arts du spectacle & alpinisme \\
\hline & compétitions sportives & $\begin{array}{l}\text { loteries simple } \\
\text { composées ou }\end{array}$ & en général & voltige \\
\hline mots croisés & en général & à report & & \\
\hline Ludus $\hat{z}$ & & & & \\
\hline
\end{tabular}

N.B. - Dans chaque colonne verticale, les jeux sont classés très approximativement dans un ordre tel tel que l'élément paidia décroisse constamment, tandis que l'élément ludus croît constamment. 